\title{
CLINICAL AND BACTERIOLOGICAL PROFILE OF EARLY ONSET NEONATAL SEPSIS ADMITTED IN NICU OF TERTIARY HOSPITAL IN BIRATNAGAR
}

\author{
Giri $A^{1^{*}}$, Sah $V K^{1}$, Poudel $B S^{2}$, Niraula $N^{2}$, Sedai $R^{3}$
}

\section{Affiliation}

1. Assistant Professor, Department of Pediatrics, Nobel Medical College Teaching Hospital, Biratnagar

2. Final year Resident, Department of Pediatrics, Nobel Medical College Teaching Hospital,Biratnagar

3. Consultant Pediatrician, Narayani Zonal Hospital, Hetauda

\section{ARTICLE INFO \\ Article History \\ Received : 28January, 2018 \\ Accepted : 21 March, 2018 \\ Published : 30 April, 2018}

(C) Authors retain copyright and grant the journal right of first publication with the work simultaneously licensed under Creative Commons Attribution License CC - BY 4.0 that allows others to share the work with an acknowledgment of the work's authorship and initial publication in this journal.

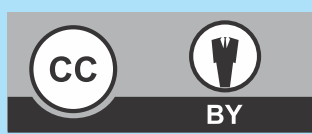

ORA 59

DOI: http://dx.doi.org/10.3126/bjhs.v3i1.19764

\section{* Corresponding Author}

Dr. Arun Giri

Assistant Professor

Department of Pediatrics

Nobel Medical College Teaching Hospital, Biratnagar

Email :drarungiri1977@gmail.com

https://orcid.org/0000-0002-7471-3164

\section{Citation}

Giri A, Sah VK, Poudel BS, Sedai R, Niraula N. Clinical and Bacteriological Profile of Early Onset Neonatal Sepsis Admitted in NICU of Tertiary Hospital in Biratnagar. BJHS 2018;3(1)5 : 370 - 376.

\begin{abstract}
Introduction

Neonatal sepsis is one of the major causes of neonatal morbidity and mortality especially in developing countries. The clinical signs and symptoms of neonatal sepsis are non specific and blood culture report is considered gold standard for confirmation of neonatal sepsis. Organisms and their sensitivity pattern vary from place to place. The confirmation of diagnosis and management of neonatal sepsis is challenging and time consuming.
\end{abstract}

\section{Objective}

The aim of this study was to find incidence of blood culture proven sepsis in suspected early onset neonatal sepsis, find out sensitivity pattern of isolated organism and to find association of risk factors and clinical signs and symptoms with blood culture proven sepsis.

\section{Methodology}

Prospective study was conducted in Nobel Medical College, Biratnagar from November 2016 to November 2017. Sample size was calculated to be 300 and blood culture was sent of each neonates admitted with suspected early onset neonatal sepsis before giving neonates with first dose of antibiotics and report of 72 hours was taken into consideration.

\section{Results}

Out of 300 cases of suspected early onset neonatal sepsis $70.3 \%$ presented with lethargy, followed by other symptoms like poor feeding, respiratory distress, fever, hypothermia, feeding intolerance, abnormal body movement and abdominal distension. Low birth weight neonates, preterm neonates, prolonged duration of per vaginal leaking and low platelets count were significantly associated with blood culture proven sepsis in this study. Incidence of blood culture positive sepsis in suspected early onset neonatal sepsis was $27 \%$. Coagulase negative Staphylococcus aureus(21\%) was predominant organism isolated followed by Klebsiella Pneumonia, Pseudomonas, Escherichia coli. All of the isolated Klebsiella and Pseudomonas and $86 \%$ of Escherichia coli were found to be resistant to ampicillin. All isolated Coagulase negative Staphylococcus aureus were sensitive to vancomycin.

\section{Conclusion}

Coagulase negative Staphyloccus aureus was predominant organism detected but majority of organisms were gram negative organisms. High resistance to ampicillin was found and cefotaxime was also less sensitive to isolated organism. Vancomycin was found to be sensitive to all isolated Staphylococcus aureus and coagulase negative Staphylococcus aureus. Amikacin was highly sensitive among causative organisms isolated.

\section{KEY WORDS}

Antibiotics sensitivity, blood culture, earlyonset neonatal sepsis 


\section{INTRODUCTION}

Neonatal sepsis (NS) is a serious bacterial infection in bloodstream in neonate at the age equal to or less than 28 days of life which is manifested by systemic signs and symptom of infection. ${ }^{1}$ Neonatal infection account for a significant cause of death in first week of life. ${ }^{2}$ Case fatality rate associated with possible bacterial infection in neonates was $9.8 \%$. It is estimated that 3.5 million neonates are of possible bacterial infection in South Asia. ${ }^{3}$

The clinical presentation of neonatal sepsis are non-specific and includes: fever, respiratory distress, lethargy, impaired or refusal of feeding, jaundice, absent Moro reflex, hypothermia, convulsions, bleeding disorder and bulging fontanel. ${ }^{4}$

Neonatal sepsis is of two types. Early onset neonatal sepsiswhen features of sepsis appear within first 72 hours and late onset sepsis- when features of sepsis appear beyond 72 hours. ${ }^{5}$ Early onset neonatal sepsis is acquired from maternal genital tract microorganisms and late onset sepsis is hospital acquired. ${ }^{6}$

Globally neonatal sepsis is one of the most important causes of morbidity and mortality among neonates during the neonatal period (0-28 day). Out of 5.9 million child deaths in 2015, almost 1 million occur in the first day of life and close to 2 million occurs in the first week of life. ${ }^{3}$ The main causes of neonatal deaths were preterm birth complications (35\%), intrapartum related complications (24\%), and sepsis (15\%) globally. According to 2011 UNICEF report, neonatal deaths accounted for $52 \%$ of all under five child mortality in South Asia, 53\% in Latin America and Caribbean and $34 \%$ in sub-Saharan Africa. ${ }^{8}$ According to global health observatory data report of 2015 approximately 4.5 million(75\%) of all under five mortality occurred within first year of life which was highest in Africa and accounted for 55/1000 live birth but was greater than five times higher than that of Europe (10/1000) live birth. ${ }^{9}$ In developing countries, neonatal mortality rate per 1000 live birth from all causes was about 34 of which most deaths occurred in the first week of life, mostly on first day of life. ${ }^{10}$

As per Nepal demographic health survey (NDHS), 2016 neonatal mortality rate in Nepal was 21 deaths per 1000 live birth. ${ }^{11}$

Antibiotics are the mainstay of treatment of sepsis. Antibiotics resistance has become challenge to the management of both early onset and late onset neonatal sepsis. ${ }^{12} 77 \%$ of isolated organism in neonatal sepsis were multidrug resistance, $60 \%$ of gram positive bacteria and $83.4 \%$ of gram negative bacteria) and nearly $80 \%$ of mortality was caused by multidrug-resistant organisms. ${ }^{13}$ Prolonged antibiotics therapy is associated with antimicrobial resistance and increased mortality in preterm infants. ${ }^{14}$

There is huge burden of neonatal sepsis and is one of the important causes of neonatal death. Antibiotics resistance has become alarming problem as per World Health Organization. To prevent antibiotics resistance as well as treat neonatal sepsis it is important to know common organisms and susceptibility pattern of antibiotics. Current study aim is to find pathogens and their antibiotics resistance pattern so that we can choose empirical antibiotics without any delay.

\section{METHODOLOGY}

This is a prospective cross-sectional hospital based study conducted in Nobel Medical college Teaching Hospital and Research Centre located in Khanchanbari, Biratnagar, Nepal from November 2016 to November 2017. After ethical clearance from the institutional review committee, data was collected from 300 newborns admitted to the department of pediatrics by means of interview with the parents and obstetricians, clinical examination and laboratory report tracing and entered into a Performa. The sample size was calculated based on a similar research conducted in Nepal Medical College Teaching Hospital, Kathmandu, Nepal. ${ }^{15}$ Taking into account the prevalence of early onset sepsis in previous study, sample size was determined and first 300 neonates fulfilling the inclusion criteria were included in the study. ${ }^{15}$ Neonates with suspected early onset sepsis born in Nobel Medical College, Biratnagar and delivered outside arriving before 72 hours of life and who had not received prior antibiotics were included in the study and neonates with gross congenital anomalies, birth weight less than 1000 grams and those who had already received antibiotics before drawing blood samples were excluded from the study.

Diagnosis of early onset neonatal sepsis was made based on the presence of symptoms, septic screening and presence of risk factors. Clinical sepsis was considered if one or more of the following symptoms were present-respiratory distress, abdominal distension, and lethargy, feeding intolerance, convulsions and temperature instability. ${ }^{16}$ Septic screening included total leukocyte count (TLC), absolute neutrophil count (ANC), C-reactive protein and platelets count. ${ }^{17}$ Risk factors considered for early onset neonatal sepsis were low birth weight (<2500 grams) or prematurity, febrile illness in the mother with evidence of bacterial infection within 2 weeks prior to delivery, foul smelling liquor, meconium stained liquor, rupture of membranes $>18$ hours, more than 3 vaginal examination and prolonged labor (sum of $1^{\text {st }}$ and $2^{\text {nd }}$ stage labour $>24$ hours). Early onset neonatal sepsis was suspected in presence of three or more risk factors, and presence of foul smelling liquor, presence of two or more antenatal risk factors and a positive septic screen and in strong clinical suspicion of sepsis. ${ }^{18}$ The associations between the clinical and bacteriological profile were analyzed using chi-square test. Level of significance in this study was considered $<0.05$.

The collected data was then coded and entered into Microsoft Excel and transferred to Statistical Package for Social Sciences (SPSS) version 20 for analysis. 


\section{RESULTS}

A total of 300 neonates were studied and analyzed. There was a male preponderance in our study with $57 \%(n=171)$ males and $43 \%(n=129)$ females. Twelve (4\%) neonates were of birth weight of 1000grams -1499 grams, 129(43\%) were of birth weight 1500-2499grams and 159(53\%) were of birth weight more than 2500 grams and 141 (47\%) were preterm and 159 (53\%) were term neonates (all appropriate for gestational age).

\begin{tabular}{|c|c|c|c|c|}
\hline \multirow[t]{2}{*}{ Birth weight } & \multicolumn{2}{|c|}{ Blood Culture } & \multirow[t]{2}{*}{ P value } & \multirow[t]{2}{*}{ Remarks } \\
\hline & Negative & Positive & & \\
\hline$<1500 \mathrm{gm}$ & $5(41.7 \%)$ & $7(58.3 \%)$ & & \\
\hline 1500-2499gm & $86(66.7 \%)$ & $43(33.3 \%)$ & 0.02 & Significant \\
\hline$>=2500 \mathrm{gm}$ & $127(79.9 \%)$ & $32(20.1 \%)$ & & \\
\hline
\end{tabular}

Table 2: Association of blood culture with gestational age $(n=300)$

\begin{tabular}{l|c|l|l|l}
\hline \multirow{2}{*}{$\begin{array}{l}\text { Gestational } \\
\text { Age }\end{array}$} & \multicolumn{2}{|c|}{ Blood Culture } & P value & Remarks \\
\cline { 2 - 5 } & Negative & Positive & & \\
\hline Preterm & $91(64.5 \%)$ & $50(35.5 \%)$ & & \\
\hline Term & $127(79.9 \%)$ & $32(20.1 \%)$ & 0.03 & Significant \\
\hline
\end{tabular}

Table 3: Association of duration of PV leaking with blood culture $(n=300)$

\begin{tabular}{|c|c|c|c|c|}
\hline Duration of & Bloor & alture & $P$ value & Remarks \\
\hline PV Leaking & Negative & Positive & & \\
\hline
\end{tabular}

$<18 \mathrm{hr} \quad 139(77.2 \%) 41(22.8 \%)$

\begin{tabular}{ll|l|l|l}
$\geq=18 \mathrm{hr}$ & $79(65.8 \%)$ & $41(34.2 \%$ & 0.03 & Significant
\end{tabular}
Out of 300 cases of suspected early onset neonatal sepsis $32(10.7 \%)$ had prolonged labour, 120 (40\%) had history of per vaginal leaking more than 18 hours duration, 37 (12.3\%) had history of more than 3 maternal per vaginal examinations, $70(23.3 \%)$ had history of maternal fever within 2 weeks of delivery, 33 (11\%) had history of delivery through meconium stained liquor and 42 (14\%) had history of foul smelling per vaginal discharge in mother.

Of total 300 cases of suspected early onset neonatal sepsis 82 cases $(27.3 \%)$ were found to be culture positive and among the studied risk factors, duration of labour, frequency of PV examination, maternal history of fever within 2 weeks of delivery, foul smelling liquor and meconium stained liquor had no significant associations with positive blood cultures. However birth weight, gestational age and history of PV leaking $>18$ hours were significantly associated with positive blood cultures.

Out of 300 cases of suspected early onset neonatal sepsis 211 (70.3\%) presented with lethargy, 196 (65.3\%) presented with poor feeding, $122(40.7 \%)$ with respiratory distress, 62 (20.7\%) were febrile, 54(18\%) neonates had hypothermia, 58 (19.3\%) had feeding intolerance,26(8.7\%) neonates had abnormal body movement and $12(4 \%)$ had abdominal distension. Among all the presenting symptoms, presentation with respiratory distress was significantly associated with positive blood cultures.

Of total 300 cases of suspected early onset neonatal sepsis 82 cases (27.3\%) were found to be culture positive of which, Coagulase negative Staphylococcus aureus was found in 17(21\%), Pseudomonas aerogenosa 14 (17\%), Klebsiella pneumonia 16(20\%), Escherichia coli 14(17\%), Staphylococcus aureus 10(12\%), Citrobacter fruendii 4(5\%), Acenitobacter anitratus 2(2\%) and Enterococcus faecalis 5(6\%).

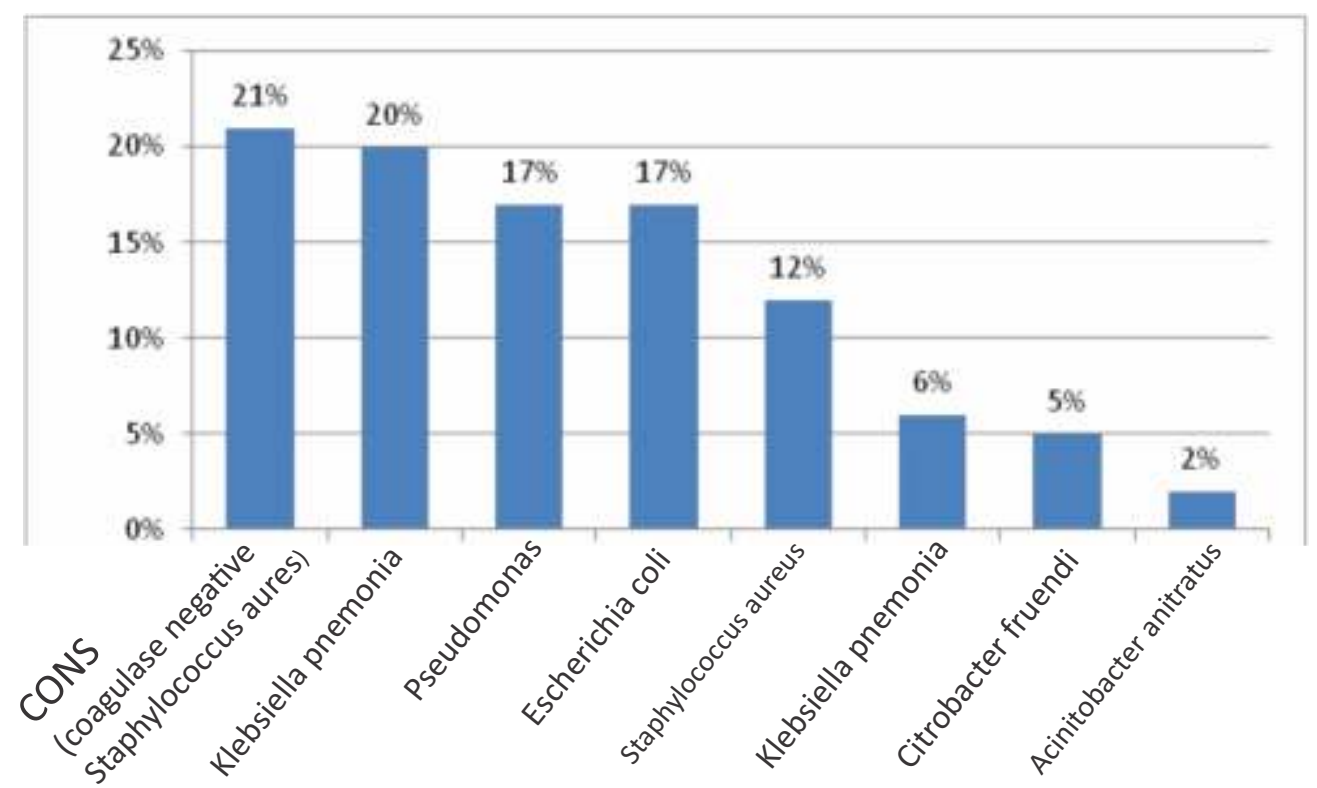

Figure 1: Distribution of detected organisms in blood culture reports $(n=82)$ 
The results of antibiotic sensitivity patterns of various isolated organisms are listed in corresponding tabular forms

Table 4: Antibiotics sensitivity patterns of Pseudomonas aeruginosa, Klebsiella pnemoniae and Escherechia coli

\begin{tabular}{|c|c|c|c|c|c|c|c|}
\hline \multirow[t]{2}{*}{ S.N. } & \multirow[t]{2}{*}{ ANTIBIOTICS } & \multicolumn{2}{|c|}{$\begin{array}{c}\text { Pseudomonas } \\
\text { aeruginosa }\end{array}$} & \multicolumn{2}{|c|}{$\begin{array}{c}\text { Klebsiella } \\
\text { pneumoniae }\end{array}$} & \multicolumn{2}{|c|}{ Escherechia coli } \\
\hline & & $\mathbf{R}$ & S & $\mathbf{R}$ & S & $\mathbf{R}$ & $\mathbf{S}$ \\
\hline 1. & Ampicillin & $14(100 \%)$ & 0 & $16(100 \%)$ & 0 & $12(86 \%)$ & $2(14 \%)$ \\
\hline 2. & Cefotaxime & $6(43 \%)$ & $8(57 \%)$ & $12(75 \%)$ & $4(25 \%)$ & $9(64 \%)$ & $5(36 \%)$ \\
\hline 3. & Ceftazidime & $2(14 \%)$ & $12(86 \%)$ & $8(50 \%)$ & $8(50 \%)$ & $6(43 \%)$ & $8(57 \%)$ \\
\hline 4. & Ciprofloxacin & 0 & $14(100 \%)$ & $5(31 \%)$ & $11(69 \%)$ & $10(71 \%)$ & $4(29 \%)$ \\
\hline 5. & Gentamicin & $2(14 \%)$ & $12(86 \%)$ & $6(37.5 \%)$ & $10(62.5 \%)$ & $3(21 \%)$ & $11(79 \%)$ \\
\hline 6. & Amikacin & 0 & $14(100 \%)$ & $6(37.5 \%)$ & $10(62.5 \%)$ & $5(36 \%)$ & $9(64 \%)$ \\
\hline 7. & $\begin{array}{l}\text { Tazobactum- } \\
\text { Pipperacillin }\end{array}$ & 0 & 14 (100\%) & $6(37.5 \%)$ & $10(62.5 \%)$ & $5(36 \%)$ & $9(64 \%)$ \\
\hline 8. & Meropenem & $4(29 \%)$ & $10(71 \%)$ & $2(12.5 \%)$ & $14(87.5 \%)$ & NA & NA \\
\hline 9. & Cefuroxime & NA & NA & NA & NA & $10(71 \%)$ & $4(29 \%)$ \\
\hline
\end{tabular}

S- Sensitive

R- Resistant

NA- not available

Table 5: Antibiotic sensitivity patterns of Citrobacter fruendi and Acinetobacter anitratus

\begin{tabular}{|c|c|c|c|c|c|}
\hline \multirow[t]{2}{*}{ S.N. } & \multirow[t]{2}{*}{ ANTIBIOTICS } & \multicolumn{2}{|c|}{$\begin{array}{l}\text { Citrobacter } \\
\text { Freundif }\end{array}$} & \multicolumn{2}{|c|}{$\begin{array}{c}\text { Acinetobacter } \\
\text { Anitratus }\end{array}$} \\
\hline & & $\mathbf{R}$ & $\mathbf{S}$ & $\mathbf{R}$ & $\mathbf{S}$ \\
\hline 1. & Ampicillin & $4(100 \%)$ & 0 & NA & NA \\
\hline 2. & Cefotaxim & $2(50 \%)$ & $2(50 \%)$ & $2(100 \%)$ & 0 \\
\hline 3. & Ceftazidime & $1(25 \%)$ & $3(75 \%)$ & 0 & $2(100 \%)$ \\
\hline 4. & Ciprofloxacin & 0 & $4(100 \%)$ & 0 & $2(100 \%)$ \\
\hline 5. & Gentamicin & $1(25 \%)$ & $3(75 \%)$ & 0 & $2(100 \%)$ \\
\hline 6. & Amikacin & $1(25 \%)$ & $3(75 \%)$ & $1(50 \%)$ & $1(50 \%)$ \\
\hline 7. & Tazobactum-Pipperacillin & $1(25 \%)$ & $3(75 \%)$ & $1(50 \%)$ & $1(50 \%)$ \\
\hline 8. & Meropenem & NA & NA & $1(50 \%)$ & $1(50 \%)$ \\
\hline
\end{tabular}

S- Sensitive

R- Resistant

NA- not available

Table 6: Antibiotics sensitivity patterns of Staphylococcus aureus, CONS and Enterococcus fecalis

\begin{tabular}{|c|c|c|c|c|c|c|c|}
\hline \multirow[t]{2}{*}{ S.N. } & \multirow[t]{2}{*}{ Antibiotics } & \multicolumn{2}{|c|}{ S. aureus } & \multicolumn{2}{|c|}{ CONS } & \multicolumn{2}{|c|}{ E. fecalis } \\
\hline & & $\mathbf{R}$ & $S$ & $\mathbf{R}$ & $S$ & $\mathbf{R}$ & $S$ \\
\hline 1. & Cloxacillin & $5(50 \%)$ & $5(50 \%)$ & $12(71 \%)$ & $5(29 \%)$ & NA & NA \\
\hline 2. & Amikacin & $1(10 \%)$ & $9(90 \%)$ & 0 & $17(100 \%)$ & $2(40 \%)$ & $3(60 \%)$ \\
\hline 3. & Linezolid & 0 & $10(100 \%)$ & 0 & $17(100 \%)$ & 0 & $5(100 \%)$ \\
\hline 4. & Teicoplanin & 0 & $10(100 \%)$ & 0 & $17(100 \%)$ & $1(20 \%)$ & $4(80 \%)$ \\
\hline 5. & Clindamycin & $7(70 \%)$ & $3(30 \%)$ & $12(71 \%)$ & $5(29 \%)$ & NA & NA \\
\hline 6. & Penicillin & $9(90 \%)$ & $1(10 \%)$ & $17(100 \%)$ & 0 & $5(100 \%)$ & 0 \\
\hline 7. & Vancomycin & 0 & $10(100 \%)$ & 0 & $17(100 \%)$ & 0 & $5(100 \%)$ \\
\hline 8. & Chloramphenicol & $2(20 \%)$ & $8(80 \%)$ & $2(12 \%)$ & 15 (88\%) & NA & NA \\
\hline 9. & Cotrimoxazole & NA & NA & $11(65 \%)$ & $6(35 \%)$ & NA & NA \\
\hline 10. & Gentamicin & NA & NA & NA & NA & $3(60 \%)$ & $2(40 \%)$ \\
\hline
\end{tabular}

S- Sensitive

R- Resistant

NA- not available 


\section{DISCUSSION}

Neonatal sepsis remains a significant cause of mortality. Diagnosis and initiation of appropriate antibiotics will definitely save life of many newborns. The bacteriological profiles of early onset neonatal sepsis are different from hospital to hospital and antibiotics sensitivity pattern also varies.

Out of 300 cases of suspected early onset neonatal sepsis, $82(27.3 \%)$ cases were blood culture proven sepses in this study whereas in study conducted in Kanti Children Hospital in $2005,14 \%$ neonates with suspected early onset Neonatal sepsis had blood culture proven sepsis. ${ }^{19}$ In another study conducted blood culture positivity among suspected early onset neonatal sepsis was found to be $25 \%$ which is similar to result observed in this study. ${ }^{20}$ In another study conducted at BPKIHS, Dharan, $20 \%$ was found to be blood culture proven early onset neonatal sepsis. ${ }^{20}$ In a study conducted in Chitwan Medical College, Bharatpur 12.6\% cases were blood culture proven early onset neonatal sepsis. ${ }^{21}$ Study conducted in Nepal Medical College Teaching Hospital, Kathmandu, $30.8 \%$ cases of suspected sepsis were blood culture proven sepsis. ${ }^{22}$ In study conducted in Dhulikhel, Kathmandu, $42.7 \%$ cases were found to be blood culture proven sepsis among suspected early onset neonatal sepsis. Hence incidence of blood culture proven sepsis is variable among various institutes.

Low birth weight, gestational age and prolonged duration of per vaginal leaking of more than 18 hours were significantly associated with blood culture positive cases. The possible reasons behind this may be babies with low birth weight or prematurity have immune dysfunction and often require prolonged intravenous access, endotracheal intubation and other invasive procedures and at 18 hours of membrane rupture, the incidence of early onset disease with group B streptococcus increases significantly. ${ }^{16}$

Regarding clinical manifestation poor feeding, lethargy, abdominal distension, feeding intolerance, temperature instability had no significant association with culture proven sepsis which was comparable to other studies with an exception of features of respiratory distress which was significantly associated with blood culture proven sepsis whereas study conducted in Dhulikhel hospital only seizure was significantly associated with blood culture proven sepsis. $^{23,25,33,34}$

Sex of neonate is not significantly associated with culture proven sepsis which is similar in study conducted in Makassar. ${ }^{24}$ In our study low birth weight and prematurity was found to be significant risk factor of neonatal sepsis which is similar to study conducted by Dr. Wahidin Sudirohusodo hospital, Makassar. ${ }^{24}$

Among organisms detected in blood culture after 72 hours, Coagulase negative Staphylococcus aureus (CONS) was seen as most common organism in $21 \%$ of neonates. In study conducted in Chitwan Medical college in 2013 Coagulase negative Staphylococcus aureus (CONS) was most predominant organism isolated(46.6\%). ${ }^{21}$ In similar study conducted in Egypt from March 2011 to August 2012 coagulase negative Staphylococcus aureus was predominant isolate in both early onset and late onset neonatal sepsis. ${ }^{23}$ In another study conducted in tertiary care Hospital in North India Coagulase negative Staphylococci (17.4\%) was predominant organism Followed by klebsiella (16.11\%) which shows similar organism pattern found in this study. ${ }^{24}$ Regarding antibiotics sensitivity pattern of CONS $29 \%$ of isolates in our study were found to be sensitive to cloxacillin whereas in study conducted in Dhulikhel, $57.1 \%$ were found to be sensitive to cloxacillin. ${ }^{25}$ In this study $100 \%$ of isolated CONS were found to be sensitive to vancomycin which is similar to result of study conducted in South India which shows sensitivity of CONS to vancomycin to be $100 \% \cdot{ }^{26}$ Also sensitivity to amikacin was found to be $100 \%$ in this study and in study at Dhulikhel sensitivity to amikacin was found to be $88.9 \% .{ }^{25}$ In this study all isolates of CONS were found to be resistant to ampicillin and in study conducted in Dhulikhel $40 \%$ of isolates were found to be resistant to ampicillin. ${ }^{25}$

Second common organism was Klebsiella pneumonia which accounted for $20 \%$ of isolates. In similar study conducted Klebsiella species accounted for $25 \%$ isolates. ${ }^{27}$ In another study conducted in Nepal Medical College Teaching Hospital, Kathmandu, Klebsiella pneumoniae accounted for $20 \%$ of isolates. ${ }^{15}$ Regarding antibiotics sensitivity pattern $25 \%$ of isolates were found to be sensitive to cefotaxime whereas in study conducted in Dhulikhel only $5 \%$ of isolates were found to be sensitive to cefotaxime. ${ }^{25} 62.5 \%$ of isolates were sensitive to amikacin in this study whereas, study conducted in Dhulikhel showed sensitivity of $88.9 \%$ to amikacin. ${ }^{25}$ In this study $87.5 \%$ of isolates were sensitive to meropenem whereas study conducted in Dhulikhel , 100\% of Klebsiella pneumonia were found to be sensitive to meropenem. ${ }^{25}$

Pseudomonas species was isolated in $17 \%$ of neonates. In study conducted in Nepal Medical College Teaching Hospital Pseudomonas was isolated in $13.4 \%$ of blood culture proven sepsis. ${ }^{15}$ In study conducted in Peshawar ,Pakistan Pseudomonas was isolated in $13 \%$ of blood culture proven sepsis. In this study $100 \%$ cases were found to be sensitive to amikacin, tazobactum-pipperacillin and ciprofloxacin and $57 \%$ were found to be sensitive to cefotaxime, and $71 \%$ were sensitive to meropenem. In similar study conducted in Rama Medical College $100 \%$ sensitivity was seen with Meropenem and amikacin and $66.67 \%$ were found to be sensitive to cefotaxime and tazobactum-pipperacillin. ${ }^{28}$ In our study study $86 \%$ of isolates of Pseudomonas were found to be sensitive to ceftazidime which was similar to study conducted in MR Medical College, Karnataka which showed sensitivity of $84.6 \%{ }^{26}$ 
Escherichia coli was isolated in $17 \%$ of neonates. Regarding sensitivity pattern $14 \%$ of isolates were found sensitive to ampicillin which is similar to results of study in Gujarat, India which shows $16.6 \%$ of isolates were sensitive to ampicillin while in another study conducted in Paropakar Maternity and Women Hospital $42.85 \%$ of isolates were sensitive to ampicillin. ${ }^{30,31}$ In this study $64 \%$ of isolates were found to be sensitive to amikacin and $79 \%$ were found to be sensitive to gentamicin whereas in study conducted in Paropakar Maternity and Women Hospital $42.85 \%$ of isolates were found sensitive to both amikacin and gentamicin. ${ }^{31} 33.3 \%$ and $16.6 \%$ of isolates were sensitive to amikacin and gentamicin respectively in study conducted in MP Shah Government College , Jamnagar, Gujarat, India. ${ }^{30}$ twenty nine percent of isolates were found sensitive to ciprofloxacin which is similar to study conducted in Gujarat, India which accounted for $28.57 \%$ isolates being sensitive to ciprofloxacin. $^{30}$

Staphylococcus aureus was isolated in $12 \%$ of blood culture proven early onset neonatal sepsis. Similar study conducted showed Staphylococcus aureus accounted for $18 \%$ of blood culture proven sepsis. ${ }^{27}$ In our study $50 \%$ cases were sensitive to Cloxacillin but studied conducted at Dhulikhel showed In our study of $91.7 \%$ to cloxacillin and study conducted in Baylesa state, Nigeria showed sensitivity of $40 \%{ }^{15,25}$ In this study $90 \%$ of isolates were found sensitive to amikacin and $100 \%$ were sensitive to vancomycin, similar pattern of sensitivity was seen in study conducted in Dhulikhel. $^{25}$.

Enterococcus faecalis was isolated in $6 \%$ of neonates in blood culture proven sepsis.In similar Study conducted in Nigeria $8 \%$ of isolates were Enterococcus species among blood culture proven sepsis. All of them were sensitive to vancomycin, linezolid, similar sensitivity pattern to vancomycin were found in study conducted in Dhulikhel hospital, and Kanpur. ${ }^{25,32}$ In this study $60 \%$ were sensitive to Amikacin but $100 \%$ sensitivity was seen in study of Dhulikhel and Kanpur.

Acinitobacter anitratus was isolated in $2 \%$ of neonates in blood culture proven sepsis. In study Conducted in Chitwan Medical College, Bharatpur, Acinetobacter species accounted for $9.5 \%$ of blood culture proven sepsis. ${ }^{21}$ Regarding sensitivity pattern $100 \%$ of isolates were found to be sensitive to ciprofloxacin in this study but study conducted in Dhulikhel showed sensitivity to ciprofloxacin to be $25 \% .50 \%$ isolates were sensitive to amikacin and

\section{REFERENCES}

1. Elsadig Yousif Mohamed S, Mohamed Ahmed A, Gadir Elimam , Sawsan M. Abdalla,, Khamis AA. Neonatal sepsis in a General Sudanese Teaching Hospital, Sudan. Int J Pharm Med Res. 2015;3(1): 177-9.

2. Baqui AH, Darmstadt GL, Williams EK, Kumar V, Kiran TU, Panwar D, et al. Rates, timing and causes of neonatal deaths in rural India: implications for neonatal health programme. Bulletin of the World Health Organization. 2006; 84(9):706-13. meropenem in this study but study conducted in Dhulikhel $20 \%$ of isolates were sensitive to amikacin and $100 \%$ were sensitive to meropenem. ${ }^{25}$

\section{CONCLUSION}

The incidence of blood culture proven sepsis in suspected early onset neonatal sepsis was nearly one out of four. The most common organism isolated was coagulase negative Staphylococcus aureus followed by Klebsiella pneumonia, Pseudomonas, Escherichia coli, Staphylococcus aureus, and Enterococcus. Overall incidence of gram negative organism was higher compared to gram positive organisms.

Low birth weight neonates, pretern neonates, prolonged duration of per vaginal leaking was found to be significantly associated with blood culture proven sepsis in this study.

Ampicillin was found to be resistant in most of isolated organisms and multidrug resistance was seen with isolated organisms. Cefotaxime was found to be resistant in gram negative organisms and cloxacillin was also found to be resistant to significant proportions of gram positive organisms.

\section{RECOMMENDATIONS}

With extensive use of antibiotics, emergence of resistance to various recommended antibiotics in early onset neonatal sepsis is seen in this study. Even resistance is seen with those antibiotics which were thought to be drugs of choice against those organisms. So antibiotics should be used judiciously and protocol for antibiotics use in different institute should be made on the basis of organisms and sensitivity pattern. Antibiotic stewardship should be used effectively.

\section{LIMITATIONS}

This study did not include anaerobic organism as culture media for anaerobic organism was found to be costly and incidence of anaerobic organism was found to be relatively very less compared to aerobic organism.

\section{ACKNOWLEDGEMENT}

We would like to acknowledge all patients and their parents, doctors and microbiologists, for their support, time and participation, whose contributions made this study possible.

\section{CONFLICT OF INTEREST}

We declare no conflict of interest.
3. Seale AC, Blencowe $H$, Manu AA, Nair H, Bahl R, Qazi SA, et al. Estimates of possible severe bacterial infection in neonates in subSaharan Africa, south Asia, and Latin America for 2012: a systematic review and meta-analysis. The Lancet Infectious diseases. 2014; 14(8):731-41. DOI: 10.1016/S1473-3099(14)70804-7.

4. Omer Saeed Magzoub A. Clinical presentation of neonatal sepsis in pediatric ward at Khartoum North Teaching Hospital, Sudan. Basic Research Journal of Medicine and Clinical Sciences. 2015;4(4):11620. 
5. Basu S, Dewangan S, Shukla RC, Anupurva S, Kumar A. Cerebral blood flow velocity in early-onset neonatal sepsis and its clinical significance. European journal of pediatrics. 2012; 171(6):901-9. DOI: 10.1007/ s00431-011-1643-y.

6. Hammoud MS, Al-Taiar A, Thalib L, Al-Sweih N, Pathan S, Isaacs D. Incidence, etiology and resistance of late-onset neonatal sepsis: a five-year prospective study. Journal of pediatrics and child health. 2012; 48(7):604-9. DOI: 10.1111/j.1440-1754.2012.02432.x.

7. Levels and Trends in Child Mortality. published in United Nations Inter-agency Group for Child Mortality Estimation. Report 2015.

8. UNICEF.org/files/UNICEF. .Levels and trends in child mortality. 2012.

9. WHO Pocketbook of hospital care for children. 2015.

10. Costello BA, et al. The state of the world's newborns. Washington. Save the Children Fund. 2001.

11. Key Indicators of Nepal Demographic and Health Survey 2016. Public Health Perspective Nepal. 2016.

12. Mohsen L, Ramy N, Saied D, Akmal D, Salama N, Abdel Haleim MM, et al. Emerging antimicrobial resistance in early and late-onset neonatal sepsis. Antimicrobial resistance and infection control. 2017; (6):63. DOI: 10.1186/s13756-017-0225-9.

13. Awad HA, Mohamed MH, Badran NF, Mohsen M, Abd-Elrhman AS. Multidrug-resistant organisms in neonatal sepsis in two tertiary neonatal ICUs, Egypt. The Journal of the Egyptian Public Health Association. 2016; 91(1):31-8.

14. Grant $\mathrm{CH}$, Arnott A, Brook T, Horne A, Hurst W, Kelly S, et al. Reducing Antibiotic Exposure in Suspected Neonatal Sepsis. Clinical pediatrics. 2017; 57(1):76-81. DOI: 10.1177/0009922816689673.

15. Shrestha RK RS, Khanal LK,Manda PK. Bacteriological study of neonatal sepsis and antibiotic susceptibility pattern of isolates in Kathmandu, Nepal. Nepal Med Coll J. 2013; 15(1):66-70. PMID: 24592800.

16. Cloherty JPE ECS, Ann R. Manual of Neonatal Care. edition 2008.

17. Da Silva KC. Accuracy of leukocyte indices and C-reactive protein for diagnosis of neonatal sepsis: a critical review. Pediatr Infect Dis J 1995(14):362-6. PMID: 7638010.

18. M. Jeeva Sankar AKD, Vinod K Paul. Sepsis in the Newborn. Delhi: CBS Publishers, Delhi. 2014.

19. Chapagain RH, Acharya R, Shrestha N, Giri BR, Bagale BB, Kayastha M. Bacteriological Profile of Neonatal Sepsis in Neonatal Intermediate Care Unit of Central Paediatric Referral Hospital in Nepal. Journal of Nepal Health Research Council. 2015; 13(31):2058. PMID: 27005713.

20. PS, DB, BN, JD, DB, SA, et al. Clinical and Bacteriological Profiles of Blood Culture Positive Sepsis in Newborns. Journal of Nepal Paediatric Society. 2007;1(2):117-120. DOI: http://dx.doi.org/ 10.3126/jnps. v27i2.1411.

21. Ansari S, Gautam R, Shrestha $S$, Neopane $P$ et al. Neonatal Septicemia in Nepal: Early-Onset versus Late-Onset. International Journal of Pediatrics. 2015;(15): DOI: 10.1155/2015/379806.
22. Shrestha RS, Khanal LK,Manda PK. Bacteriological study of neonatal sepsis and antibiotic susceptibility pattern of isolates in Kathmandu, Nepal. Nepal Med Coll J. 2013;15(1):71-73.

23. Shehab EI-Din EM, El-Sokkary MM, Bassiouny MR, Hassan R. Epidemiology of Neonatal Sepsis and Implicated Pathogens: A Study from Egypt. BioMed research international. 2015. DOI: 10.1155/ 2015/509484.

24. Lamba M, Sharma R, Sharma D, Choudhary M, Maheshwari RK. Bacteriological spectrum and antimicrobial susceptibility pattern of neonatal septicaemia in a tertiary care hospital of North India. The journal of maternal-fetal \& neonatal medicine : the official journal of the European Association of Perinatal Medicine, the Federation of Asia and Oceania Perinatal Societies, the International Society of Perinatal Obstet. 2016;29(24):3993-8.

25. Shrestha S SN, Dongol Singh S, Shrestha RPB, Kayestha S, Shrestha M, Thakur NK2. Bacterial Isolates and its Antibiotic Susceptibility Pattern in NICU. Kathmandu university medical journal. 2013; 11(41):66-70. PMID: 23774417.

26. Kumaravel K S RB. A Study of the Bacteriological Profile and Antibiotic Sensitivity in Neonatal Septicemia. International journal of Contemporary Medical Research. 2016;3(6):343-7.

27. Khan AKM. Pathogens associated with sepsis in newborns and young infants in developing countries. The Pediatric Infectious Disease Journal. 2009;28(1):S10-8.

28. Ullah O, Khan A, Ambreen A, Ahmad I, Akhtar T, Gandapor AJ, et al. Antibiotic Sensitivity pattern of Bacterial Isolates of Neonatal Septicemia in Peshawar, Pakistan. Archives of Iranian medicine. 2016;19(12):866-9.

29. Sharma CM, Agrawal RP, Sharan H, Kumar B, Sharma D, Bhatia SS "Neonatal Sepsis": Bacteria \& their Susceptibility Pattern towards Antibiotics in Neonatal Intensive Care Unit. Journal of clinical and diagnostic research : JCDR. 2013;7(11):2511-3.

30. Hardik V Vaniya NMP, Jitendra M Agrawal, Hiren R Trivedi , Jatin V Dhanani, Jayesh D Balat1. Antimicrobial culture sensitivity pattern in neonatal sepsis in a tertiary-care hospital International Journal of Medical science and Public Health. 2015;5(04).

31. Khanal R1 MS, Acharya GP3. Bacteriological Profile of Neonatal Sepsis in a Tertiary Level Hospital of Nepal. J Nepal Paediatr Soc. 2014;34(3):205-8. DOI: http://dx.doi.org/10.3126/jnps.v34i3.9183.

32. Chandra Madhur Sharma RPA, Hariom Sharan. Neonatal Sepsis: Bacteria \& their Susceptibility Pattern towards Antibiotics in Neonatal Intensive Care Unit. Journal of clinical and diagnostic research: DOI: 10.7860/JCDR/2013/6796.3594.

33. Shrestha S, Dongol Singh S, Shrestha NC, Shrestha RPB, Madhup SK2 Comparision of Clinical and Laboratory Parameters in Culture Proven and Unproven Early Onset Sepsis in NICU. Kathmandu university medical journal.2013;11(44):310-4. PMID: 24899326. 